\title{
REACCIONES LEUCEMOIDES E HIPERLEUCOCITOSIS EN CLINICA INFANTIL
}

\author{
Drs. ALFONSO COSTA y ROSA VOLOSKY \\ Citedra de Pediatria del Prof. Arturo Scroggie y Laboratorio de Hematologia del \\ Hospital "Roberto del Fio".
}

\section{Introducción.}

Se observan en clínica infantil, leucocitosis elevadas con mucho mayor frecuencia que en adultos. La delimitación de estos cuadros hematológicos, su relación con la clínica, y su significado mismo nos han parecido dignos de estudio por tratarse de una particularidad de la hematología pediátrica.

El objeto de este trabajo es mostrar las diversas características, tanto clinicas como hematológicas de estas alteraciones, $y$ corresponden a lo que hemos observado en los últimos 5 años.

Conocida ya desde los tiempos de Virchow' la leucemia, caracterizada en aquella época fundamentalmente por un elevado aumento del número de leucocitos en la sangre, se tendió a considerar como tal enfermedad, a todas aquellas que presentaban un gran número de glóbulos blancos en la circulación.

Pero la observación de casos con altas leucocitosis, $\sin$ los demás caracteres de la leucemia obligó a separar estos últimos cuadros de los primeros.

Parecen haber sido Gilbert y Weil ", en 1900 , los primeros que señalaron esta circunstancia en pacientes con infecciones amigdalianas. Luego se la observó en otros cuadros infecciosos.

Las descripciones iniciales se referían a altas leucocitosis con células de tipo mieloide. Las leucocitosis de tipo linfoide fueron señaladas por Jackson y Smith ${ }^{3}$ en 1915.

Las elevadas eosinofilias de ciertos cua dros clínicos, plantearon la existencia de Ia lencemia eosinofílica como variedad de la mieloide, pero pronto se pudo comprobar que algunos de estos enfermos tenian una evolución favorable y que, por lo tanto, no cabían dentro del cuadro de las leucemias. Las primeras reacciones de tipo eosinofilico fueron descritas por Giffin * en 1919, y también por Shapiro "en el mismo año. Su estudio ha sido reactualizado a raíz de las comunicaciones de Loef- fler " en 1932 y de Valledor y sus colaboradores 7.3 .4 de 1939,1942 y 1943.

El término de reacción leucemoide para estos cuadros clínicos hematológicos, fué introducido por Krumbhaar ${ }^{10}$ en 1926. Pero para el caso de las reacciones eosinofilicas ha encontrado aceptación el término de "Leucemoide Eosinófilo".

Personalmente hemos dividido estos procesos en dos categorías:

a) Reacciones leucemoides, y

b) Hiperleucocitosis.

Entendemos por "Reacción Leucemoide" el aumento considerable y transitoris del número de leucocitos sanguíneos, asociado a la presencia de células inmaduras, sin las restantes características hematológicas o clínicas propias de la leucemia.

Definimos como "Hiperleucccitosis" aquellos casos en que existe un aumento considerable de leucocitos sanguíneos, sin células inmaduras ni las restantes caraeterísticas propias de la leucemia.

No existe un límite natural para demarcar el número de leucocitos que separan una leucocitosis banal de una hiperleucocitosis. Arbitrariamente hemos puesto esta separación en 50.000 leucocitos.

Consideramos reacciones leucemoides aquellos que presentaron más de $5 \%$ de células inmaduras. Ya que es frecuente observarlas en escasa proporción en diversas enfermedades del niño, y como éste además, reacciona fácilmente con altas leucocitosis, los límites fijados por nosotros son arbitrarios y evidentemente que otros podrán tener distinto criterio para clasificar un cuadro hematológico como simple leucocitosis o como hiperleucscitosis o como reacción leucemoide.

Tanto las reacciones leucemaides como las hiperleucocitosis podemos subdividirlas según el tipo de células que predominan en la sangre circulante. Si estas son linfoides, tendremos las hiperleucocitosis linfoides o las reacciones leucemoides linfoides. Si encontramos predominio de células mieloides, hablaremos de hiperleucocitosis mieloide o de reacción leucemoide 
TAB1A NOR

CLASIFICACION DE LAS REACCIONES LEUCEMOIDES E HIPERLEUCOCITOSIS

1.-Reacciones teutcinoides:

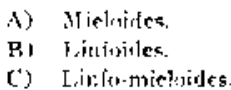

2... Hiret'eucocitosis:
A) Mieloirles.
B) limiogiles.
(') Linfo-mielosiles.

s.-Leucendide eosinofito.

mieloide. Finalmente existe buena proporción de casos en que no existe un predominio franco de células mieloides o linfoides, encontrándose ambos tipos en proporciones más o menos iguales. A éstos los hemos denominado hiperleucocitosis o reacciones leucemoides de tipo "linfo-mieloide".

Por último debemos considerar, en ciertos aspectos, como una entidad un tanto aparte, el Leucemoide eosinófilo.

Un esquema general de la clasificación de estos cuadros es la que damos en la tabla No 1.

No existen sintomas clínicos que permitan sospechar algunos de estos cuadros. Unicamente se recanoce su existencia por el examen hematológico, constituyendo por lo tanto un hallazgo de laboratorio.

No se ha encontrado una explicación satisfactoria para estas alteraciones hematológicas. Sólo se han precisado los cua-

\section{Gräfico-no 1}

Distribución por edades de los enfermas con biperlevcocitosis y reacción leucemoide.

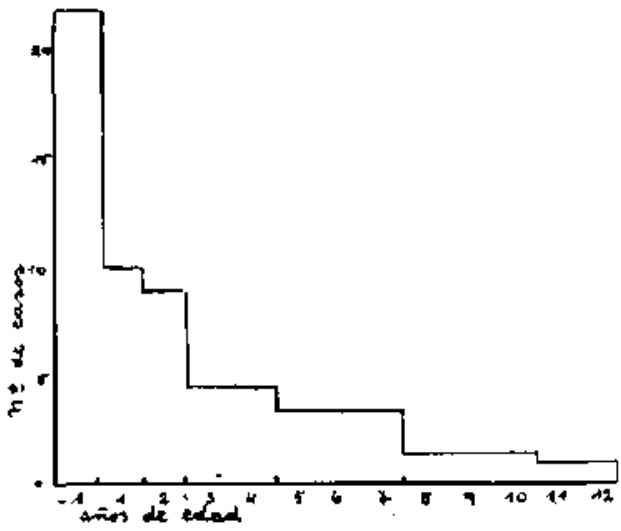

TARTA N\& 2

DISTRIBUCION POR EDADES DE LOS ENFERMOS

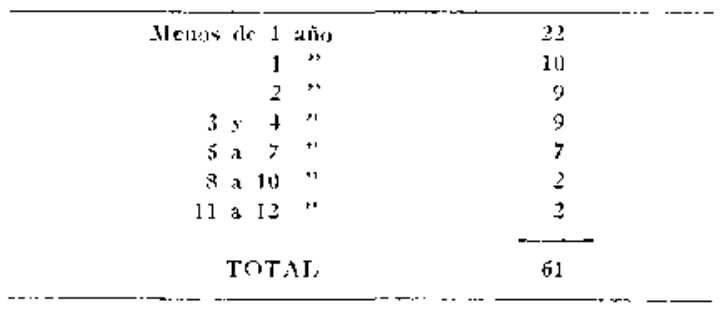

dros clínicos que más frecuentemente se acompañan de ellas.

Los más citados en la literatura y que en parte corresponden a nuestra experiencia son los siguientes:

1. Infecciones agudas: coqueluche, enterocolitis, sarampión, parotiditis, meningitis purulenta, sepsis y septicemias.

2. Infecciones crónicas: tuberculosis, lúes.

3. Anemias hemolíticas.

4. Tumores malignos.

5. Intoxicaciones.

6. Enfermedades de Hodgkin.

7. Diversos cuadros o enfermedades difíciles de clasificar.

\section{Material estudiado.}

Reunimos todos los pacientes que en los últimos 5 años (Julio de 1949 a Junio de 1954) tuvieron en su examen hematológico una reacción leucemoide o una hiperleucocitosis. Estos fueron $55 \mathrm{y}$ a ellos agregamos 5 observaciones de años anteriores que fueron controlados en el Laboratorio. Incluímos finalmente un leucemoide eosinófilo. En total presentamos 61 observaciones.

Controlamos nuestros casos en general hasta la normalización del número de leucocitos, o hasta el fallecimiento. De los que sobrevivieron, varios los hemos seguido por años después de su alteración hematológica, no comprobando en estos casos nuevas alteraciones de este tipo.

De los pacientes, 22 tenían menos de 1 año de edad y 40 eran mayores de 1 año. Un mayor detalle de la distribución por edades está dada en la tabla No 2 y en el gráfico $\mathrm{N}^{\prime} 1$.

En nuestra casuística el grupo más importante lo forman los enfermos con coqueluche que son 22 , lo que representa un $36 \%$, le siguen los enfermos de enteroco- 
TABLA No 3

CUADROS CLINICOS EN LOS ENFERMOS CON HIPERLEUCOCITOSIS O REACCION LEUCEMOIDE

(x) (1)

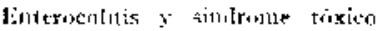

Tuleejen:lasis primaria

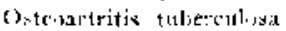

Serringitis: gingrlenta

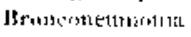

S'arice':

(i.):uculde letritis aguda

F.etitsles grijeal

["úr]nura

Mistintuclersis

Traminatismb eranearo

Q11emiaduras

Epilepria exercial

Lencontrive mosimbitilo

TOT:II,

litis $\mathrm{y}$ síndrome tóxico del lactante, que son 19 o sea un $31 \%$.

El resto, 20 pacientes ( $33 \%$ ) lo constituyen un grupo de diversas enfermedades.

En la tabla $N^{\circ} 3$ y gráfico No 2, detallamos los diversos cuadros clínicos concomitantes con estas alteraciones hematológicas.

Desde el punto de vista hematológico hemos reunido los casos en tres grandes grupos:

TABLA N* 4

DISTRIBUCION DEL NUMERO DE LEUCOCITOS

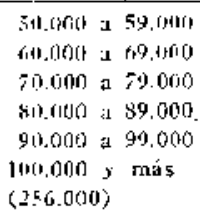

TOTAL

$\begin{array}{lr}- & 29 \\ = & 12 \\ = & 3 \\ = & 4 \\ = & 2 \\ = & 5 \\ & \end{array}$

I. Hiperleucocitosis: 46: forman un $75,5 \%$ de nuestros casos.

II. Reacciones leucemoides: 14 : representan el $23 \%$.

III. Leucemoide eosinófilo: 1 : constituye el $1,5 \%$.

Los dos primeros grupos han sido subdivididos de acuerdo con la clasificación ya planteada. Las hiperleucocitosis fueron en proporción sensiblemente iguales, ya sea mieloides (15), linfoides (13), o linfomieloide (18). De las hiperleucocitosis, 8 presentaban eritroblastos en la sangre, en proporción discreta.

Las 14 reacciones leucemoides fueron casi todas del tipo mieloide, 12 casos de un total de 14. Las otras 2 fueron de tipo linfomieloide. No observamos ninguna de tipo linfoide. Más de la mitad, 8 de 14 casos de las reacciones leucemoides mie-

$$
\begin{aligned}
& \text { Gráfico no } 2 \\
& \text { Cuadros clínicos en los enfermos con } \\
& \text { hiperleucocitosis y reacción leucemoide. }
\end{aligned}
$$

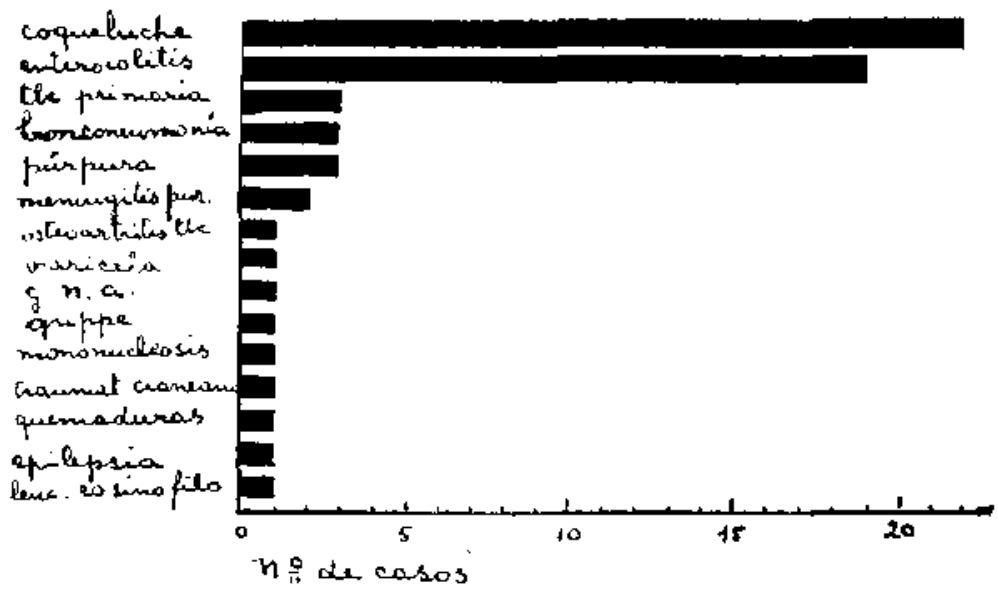




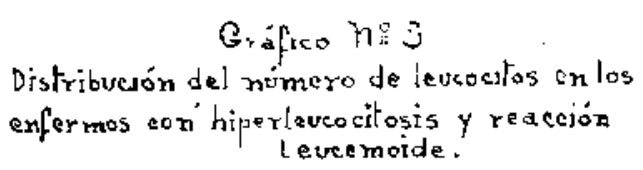

$50+59000$
$60=69.000$
$70=79.000$
$80 a 89000$
90 a 99000
100 a 286.000

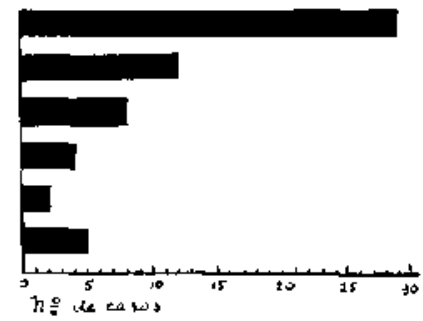

loides, tenían eritroblastos en la sangre circulante.

Considerando únicamente el tipo celular predominante y reuniendo los dos grupos, encontramos lo siguiente: el tipo mieloide 27 casos o sea un $45 \%$; de tipo linfoide 13 enfermos $(22 \%)$ y de tipo linfomieloide 20 pacientes o sea un $33 \%$.

\section{Anemia.}

Del conjunto de nuestros casos 32 tenían anemia. Esta fué leve en 21 casos ( 3 a 3,9 millones de hematies ), fué mediana 12 a 2,9 millones de hematies) en 4 casos $\mathrm{e}$ intensa en 7 casos (menos de 2 millones de glóbulos rojos).

\section{Leucocitosis.}

El número de leucocitos fluctuó entre 50.000 y $256.000 \times$ mmc., con la excepción de leucemoide eosinófilo en el que tuvi$\operatorname{mos} 16.000 \times \mathrm{mmc}$. Un detalle de la distribución del número de leucocitos está dada en la tabla número 4 y gráfico $\mathrm{N}^{\prime} 3$.

TABนA Y 5

ALteraciones hematologicas EN COQUE. LUCHE COMPLICADA DE BRONCONEUMONIA

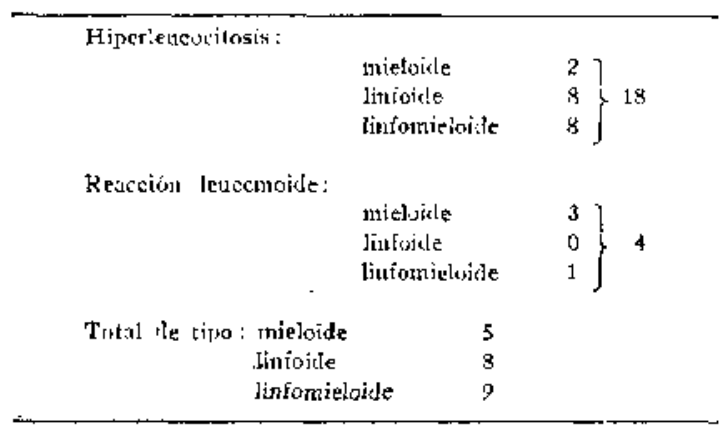

TABIA Ne G

CARACTERISTICAS HEMATOLOGICAS DE LOS ENFERMOS CON ENTEROCOLITIS Y SINDROME TOXICO DEL LACTANTE

\begin{tabular}{|c|c|c|c|}
\hline Hiperlentucilusis: & 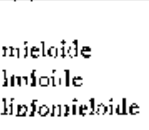 & $\left.\begin{array}{l}8 \\
2 \\
6\end{array}\right]$ & 16 \\
\hline Reacción leckernoicle: & $\begin{array}{l}\text { injelovile } \\
\text { linfojise } \\
\text { linfonsiekvide }\end{array}$ & $\left.\begin{array}{l}2 \\
1) \\
1\end{array}\right\}$ & 3 \\
\hline $\begin{aligned} & \text { Tutal de tifio: } \text { micloisle } \\
& \text { Jiufoile } \\
& \text { finfomieloi }\end{aligned}$ & ide & & \\
\hline
\end{tabular}

\section{Mielograma.}

Se hizo en 13 enfermos, 4 de ellos tuvieron mielograma normal, 4 una hiperplasia eritroblástica y 5 una hiperplasia de la serie linfoide, granulosa o indiferenciada, que podía relacionarse citológicamente con la alteración encontrada en la sangre periférica. Aunque el número de pacientes en los que se practicó mielograma es escaso, no dejó de llamarnos la atención que en 8, o sea más de la mitad, no encontrásemos alteraciones, que se correspondieran con las de la sangre periférica.

La correlación de los hallazgos clínicos con los hematológicos nos da lo siguiente:

1. Coqueluche: Se trata de 22 casos, todos complicados de bronconeumonia. Los hallazgos hematológicos están en la tabla N! 5.

Deseamos hacer notar esta característica hematológica ya que habitualmente se

TABLA N: 7

\section{HALLAZGOS HEMATOLOGICOS EN UN GRUPO DE ENFERMOS AFECTADOS DE DIVERSOS CUADROS CLINICOS}

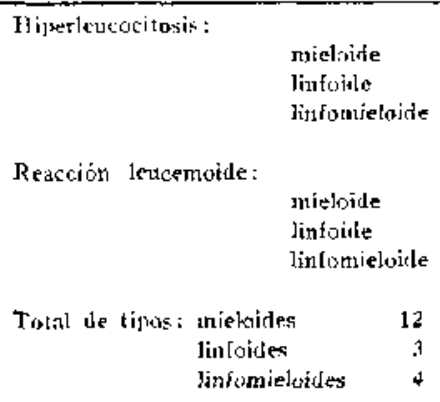


TALI.A Nं9

CANTIDAD DE LEUCOCITOS OBSERVADOS EN LOS FALLECIDOS

\begin{tabular}{|c|c|c|c|}
\hline N: de leucocitos & N"enfermos & N: fallecidos & $\%$ \\
\hline 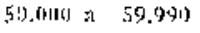 & 29 & 11 & \\
\hline 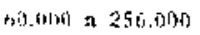 & 31 & 6 & 19 \\
\hline
\end{tabular}

acepta que la coqueluche produce reacciones de tipo linfoide.

De estos enfermos con coqueluche fallecieron 5 o sea un $23 \%$.

2. Las enterocolitis y sindromes tóxicos del lactante: Sus características hematologicas están anotadas en la tabla $N^{2} 6$.

La letalidad de este grupo fué de $7 \mathrm{ca}-$ sos, o sea $37 \%$.

Dentro de este grupo está el caso con mayor número de leucocitos de toda nuestra experiencia: es el número 4 que tiene 256.000 leucocitos $\mathrm{x}$ mmc.

3. Grupo Mixto: Está integrado por enfermos con muy diversos cuadros clínicos, de cada uno de los cuales tenemos muy pocos representantes como para que nos permitan formar un grupo homogéneo. Sus diagnósticos clínicos están ya dados en el cuadro No 3. Sólo por seguir la exposición sistemática hemos analizado los hallazgos hematológicos que se muestran en la tabla No 7.

De éstos fallecieron 5 , o sea un $20 \%$.

Con objeto de ver si era posible relacionar la gravedad del cuadro y el número de leucocitos, estudiamos la cantidad de glóbulos blancos en los fallecidos. Los resultados se aprecian en la tabla No 8 y gráfico No 4.

Contrariamente a lo que podria suponerse, una mayor leucocitosis no se acompaña necesariamente de una mayor gravedad del cuadro clínico.

\section{Comentario.}

Para explicar el mecanismo de producción de la hiperleucocitosis y reacciones Ieucemoides podemos suponer dos hipótesis:

1. Que el agente causante de la enfermedad, produzca él mismo y específicamente el pasaje exagerado de leucocitos desde los órganos hematopoyéticos hacia la sangre.

2. Que el terreno o capacidad reaccional del órgano hematopoyético, ante un estimulo cualquiera, inespecifico por lo

$$
\text { Grafico n:4 }
$$

Relacion entre el núacro de tercocitos y la letalidad en enfermos con hiparlevesitosis y reaceión leveemoide.

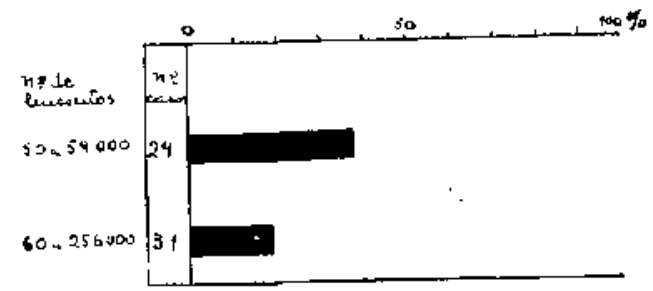

tanto, sea el que determine esta alteración.

Como $2 / 3$ de nuestras observaciones presentan como enfermedad concomitante, ya sea la coqueluche o la enterocolitis, ésto nos hace pensar que este tipo de reacción hematológica no se debe al terreno mismo, sino que acompaña de preferencia a estas dos enfermedades. Si las reacciones leucemoides e hiperleucocitosis fueran debidas primordialmente a una capacidad reaccional del tejido hematopoyético, veríamos estas alteraciones acompañando a cualquiera de las distintas enfermedades que se presentan en la infancia.

Es fácil suponer que en nuestro primer grupo de enfermos, o sea los con coqueluche, sea la acción específica del germen la que determine la alteración hematoló gica. Pero no es tan claro que sea así en las enterocolitis y sindromas tóxicos del lactante, ya que no podemos atribuír estos últimos trastornos a un solo agente etiológico y en cambio, la deshidratación y otros trastornos metabólicos que acompañan o complican este proceso es muy probable que sean los verdaderos determinantes de Ia reacción leucemoide o hiperleucocitosis.

E1 $1 / 3$ restante de nuestros casos está constituído por un grupo heterogéneo de cuadros clínicos, sin ningún nexo etjológico común. Nos sentimos por lo tanto autorizados a atribuír la respuesta sanguínea a una alteración del terreno.

Por las características encontradas en los mielogramas, a las que nos referimos anteriormente, podemos pensar que es probable que exista una alteración en el pasaje de células desde la médula hasta la sangre, y no un trastorno de la hematopoyesis propiamente dicha.

Las perturbaciones del mecanismo que regula el pasaje de leucocitos del tejido 
hematopoyético a la sangre pueden ser del tejido mieloide como del linfoide o de ambos silmultánea o sucesivamente, como lo ilustra la observación clínica $\mathrm{N}: 59$.

En el caso del leucemoide eosinófilo no encontramos, y es lo más frecuente, una causa que explique Io ocurrido. Pero del análisis de sus características nos sentimos inclinados a atribuír el cuadro a una reacción del terreno y no como una manifestación secundaria ni específica ante una noxa.

\section{Casuística.}

Presentamos los datos más importantes de todas nuestras observaciones en la tabla $N^{4}$. 9 , y a continuación algunos casos clínicos que nos han parecido más representativos.

Obs. N" f: O. M. Tieks 49/18367. Niño de 3 años de edad. qule ingresa al Hospital por "enterocolitis grave". Al examen hematológico se compruehan 250.000 kucocitos, con $71 \%$ de linfocitos, $4 \%$

TABIA NOS

DATOS MAS IMPORTANTES DE LOS ENFERMOS CON HIPERLEUCOCITOSIS $Y$ REACCIONES LEUCEMOIDES

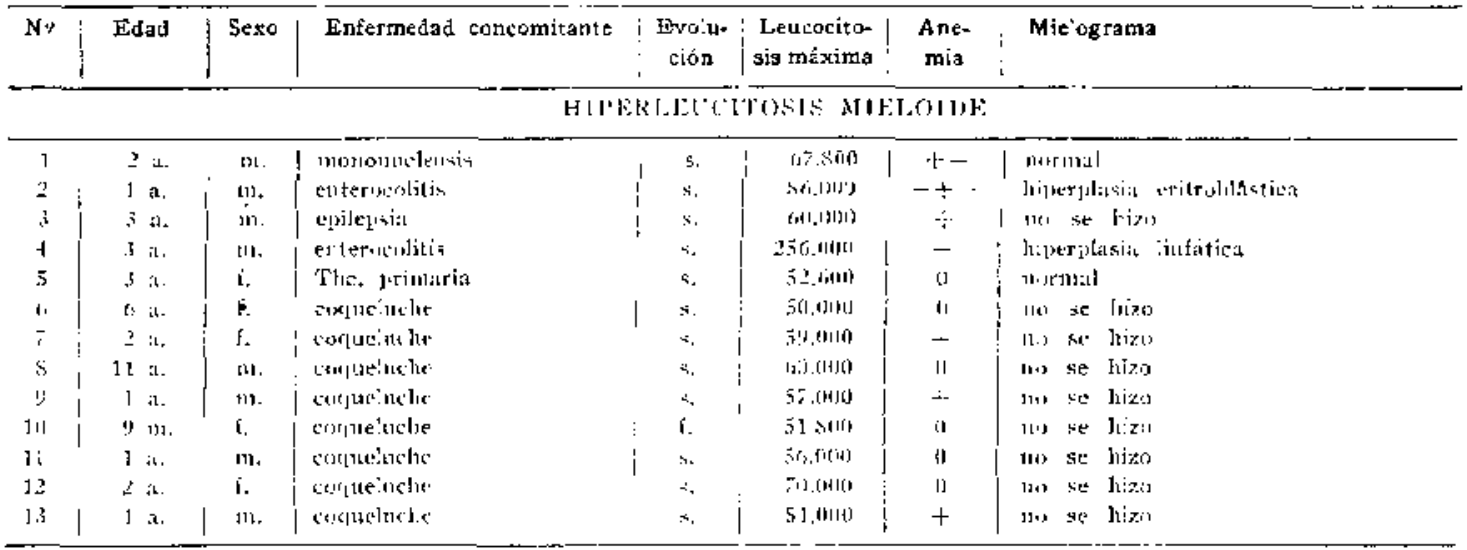

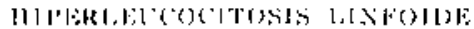

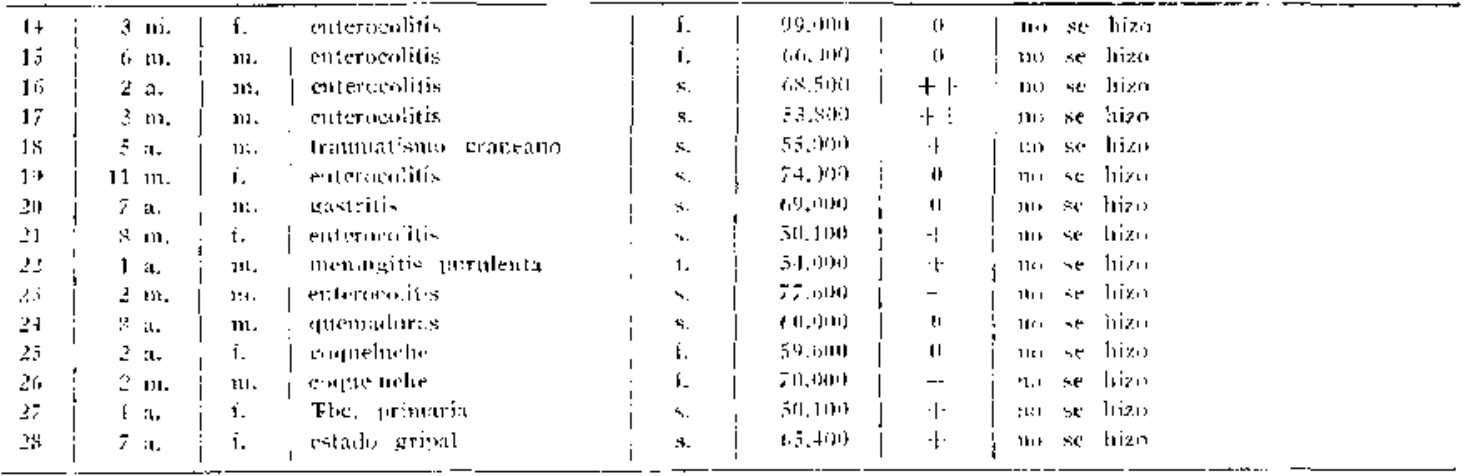

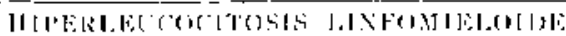

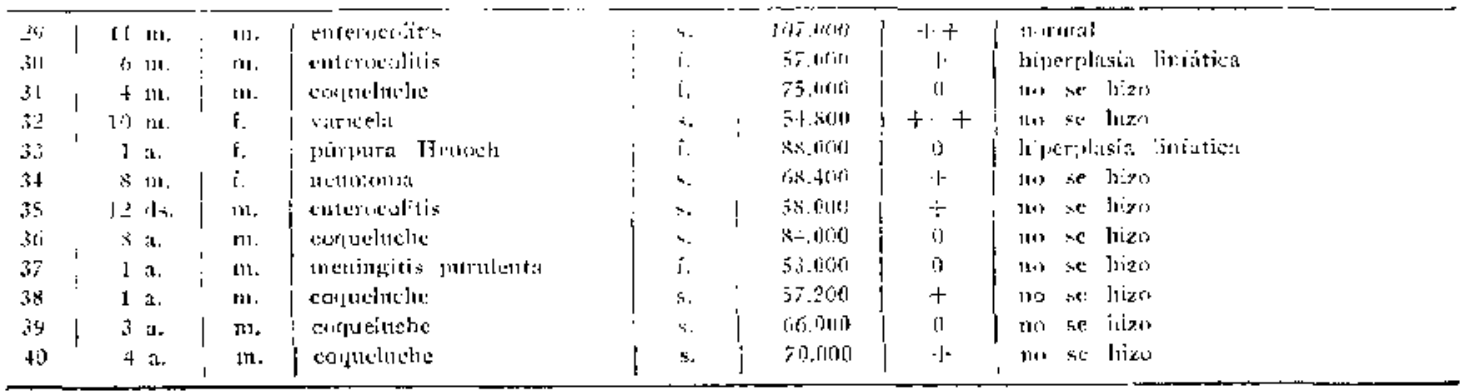




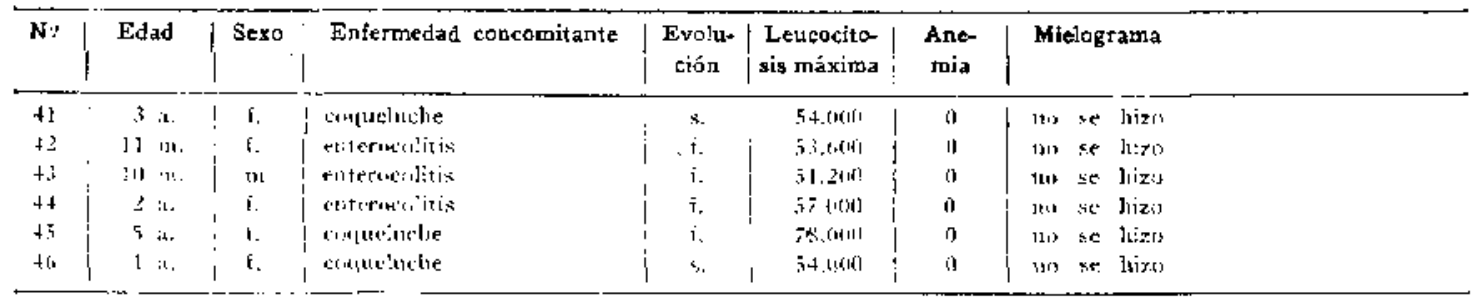

Klac?

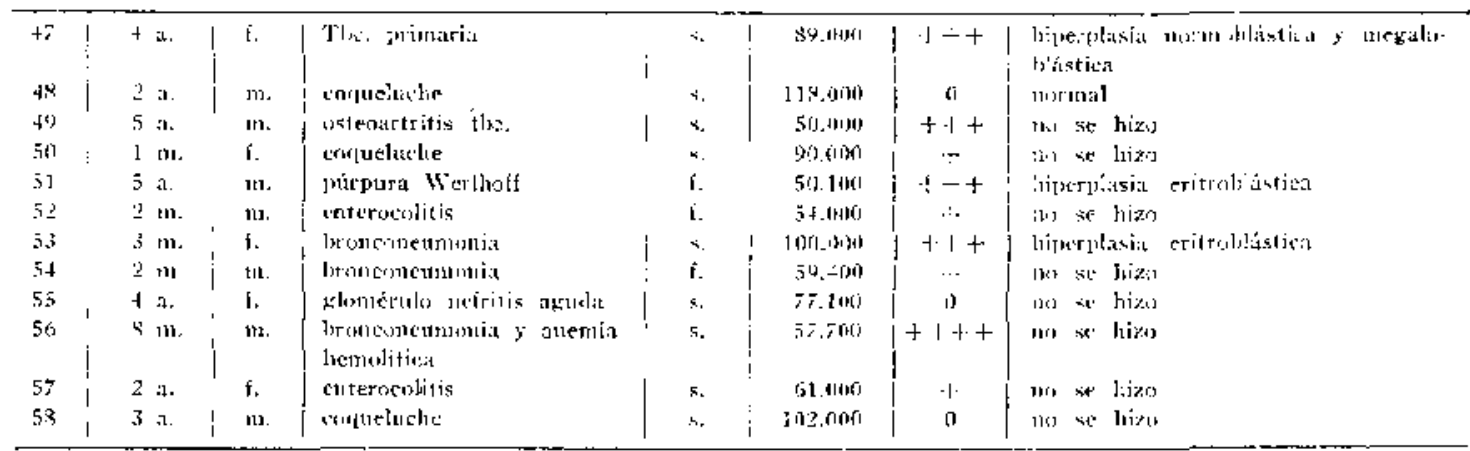

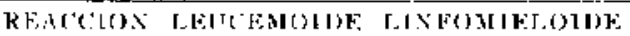

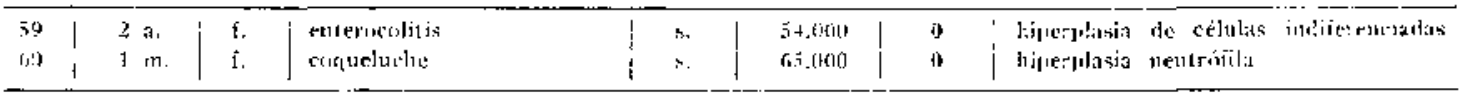

LEUCEMOIDE EOSINOFILO

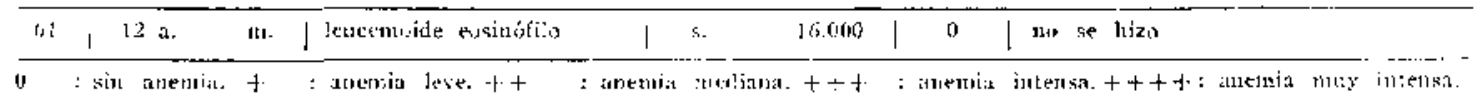

monocitos y $25 \%$ de ncutrófilos. Los gangijos liníticos. bazo a bigado. no estaban aumenudos de iamaño. Un míelograma demostró intensa hiperplasia linfárica (65\%). Se planté el diagnóstico de Jeucemia linfática. el cual fué recbazado, tanto por la crolucjón clinica, como la bematológica.

En controles diarios de hemogramas se comprobó una cifra normal de jeucociros a los 9 dias de ingreso. junto con un aumento de neutrófilos y disminución de linfoltos. Iiste enfermo fui esrudiado elinica $v$ hematolomicarnente más de 4 meses y fuć dado de jlıs. con henugrame persistentemente nurmal.

Ohs. N'. 14: J?. Ml. G. Niña de 3 meses gue ingresu por enterocolitis y sindrome lóxico. lallece pocas horas después. I.a autopsia confirma ol diagnóstico.

En el hemograma se encuentran 90.000 glóbulos blancos, con $12 \%$ de monocisos. $2+\%$ linfocitos. $46 \%$ segmentados, $13 \%$ baciliformes. $3 \%$ eosinofilos, $1 \%$ juveniles y $16 \%$ erilroblastos.

Obs. N': 35: J. M. P. Tiicha 108363 . Recita nacido primatuto, del sexo masculino que ingresi con enterocalitis y sintomas de peritonitis, que regresan en 4 dias, con tratamienro nićdico. Su primer hemograma revela 58.000 leucotitos con $7 \%$ monocitos. $30 \%$ linfocitos. $59 \%$ neutrófilos y $3 \%$ eosinófilos. Hemogramas posteriores revelan ura disminución paularina de leucocitos. que se normalizan más o menos. 3 semanas despoís del ingreso.
Obs. N +7: A, S. Ficha 1806. Nína de 4 años que ingress febrit $y$ con un sindrome bemortágico agudo. E1 bazo esti aumentado de tamaño, y por la anéria intens. $y$ el número de lcucocitos, 89.000 con mielocitos, se plancéa la posibilidad de una leucomia: pero el mielograma mucstra una intensa biperplasia etitroblástica $(50 \%$ de exitroblastos $) \sin$ aumento de la serie granulosa. ni linfálica, ni indife renciada. Con estos antecedentes, se bace el diagnóslico do reacción lencomoide micioide.

Henalologicamenle st nomaliza en 2 sconanas. canLo en la sangre, cono en ia médula. Conrroles posteriores, busta 2 meses después. no revelon nado anotmal.

Como enfermedad concomilante fincerpreada cormo desencadenamente, se comprueba un complejo primario tuberculaso en crolución.

Obs. No 59: R. M. B. Ficha 33870 . Niño d: 2 años de edad, que ingres.l, por gostroenteritis antda. acompañada de síndrome purpútico y hepsousplenomegalia. En el examen hematológico se encuentran número normal de glóbulos rojos y $\overrightarrow{\mathbf{j}}+\mathbf{4} 000$ leucocitos ron $30 \%$ de linfocitos, $16 \%$ de neurótilos, $4 \% \mathrm{mic}$ locitos y $47 \%$ celulas inmaduras e indiferenciadas. Sc plantea el diagnóstico de leucemia. El nielograma practicado el mismo dia, liende a confimar el diagnóstico, ya que se cocuenter casi $20 \%$ de células indifirenciadis. 
Hemogramas y miclogramas posteriotes, permiten descatar este diagnósrico hematológico. ya que a los 4 dias did ingreso sc comprueban 8.000 leucocitos con $60 \%$ di linfocitos $y 24 \%$ de neutrófílos. sin células blísticas en la sangte circulante. Asimismo el mielograma tambien se normaliza.

L̇ exámenes posteriores, el número de leucocitos Se mantitne notmal: pero se observa una intensa linfocicosis de mis de $80 \%$, iuto con la cortespondiente disminución de ć́lulas geanulosas (llegaron a constituir sólo el $4 \%$ de los glóbulos blancos circu. lanles. en un momento dado).

Sf ta de alts sano, con hemograma notmal.

Ohs. No 61: S. I. 12 años de edad, del sero masculine. Presenta sintomatologia de obstrucción intestinal. Un bemograma revela 16.000 leucocitos $y+0 \%$ ensinótilos. que hace pensat. en un primer momento en una triquinosis. $S_{\mathfrak{c}}$ acentúan los sintomas obstructivos. aparecen ascilis y gran perdida de peso. Se prac. lica crtonces, uлa laparotomía exploradora y se comprucba, además de la ascitis. infarto de los ganglios mesentéricos. y equimosis anulares del intestino. co. tmo si bubiera existido una invaginación transitoria, En la biopsia de un ganglio mesentérico, como también en el líquido ascítico, se encucntran abundantes cosinófilos

Coincidiendo con la laparotornia y sin otra terapéutica. mejora rápidamente.

Controlado tlínica y hematológicamente hasta 6 años después de este cuadro. se encuentra en buenas condiciones.

Sc hace el diagnóstico en este caso, de leucemoide eosinófilo.

\section{RESUMEN}

Se reúnen 61 observaciones de reacciones leucemoides, de hiperleucocitosis y leucemoide eosinófilo que corresponden a todos los casos observados en el Hospital Clínico de Niños "Roberto del Rio" durante un periodo de 5 años.

De estos enfermos, 22 tenían menos de un año y 39 un año o más.

En $33 \%$ se encontró esta alteración hematológica acompañando a la coqueluche, complicada de bronconeumonia.

$1 / 3$ de los casos tenían enterocolitis o sindrome tóxico del lactante.

El resto estaba constituido por enfermos afectados de muy distintos cuadros clínicos.

Se incluye también en esta casuística un caso de leucemoide eosinófilo.

Se separa la reacción leucemoide propiamente dicha en la cual existe hiperleucocitosis y células inmaduras (mieloi- des o linfoides o ambas) de la hiperleucocitosis (sin células inmaduras).

$75 \%$ de los casos fueron hiperleucocitosis y el resto, reacciones leucemoides.

Los valores más elevados de leucocitos fueron de $100.200,102.000,107.000$, 118.000 y 256.000 .

El mielograma no mostró en general alteraciones semejantes a las encontradas en la sangre periférica.

Se interpretan estos cuadros hematológicos en $2 / 3$ como atribuibles a la enfermedad concomitante $y$ en $1 / 3$ a una reacción del terreno.

No se encontró una relación entre el aumento del número de leucocitos y la gravedad de los casos.

\section{SUMMARY}

61 cases of leukemoid reactions and hiperleukocytosis are gathered in a period of. 5 years in the "Roberto del Rio" Children's Hospital. Out of these, $75 \%$ of cases corresponded to hiperleukocytosis and $25 \%$ to leukemoid reactions (with immature cells).

In $1 / 3$ of cases this hematologic condition was found in whooping cough complicated with bronchopneumonia, in other $1 / 3$ of cases in enterocolitis and toxic syndrome in infancy and in the remaining $1 / 3$ of cases in different other clinical pictures. One case of eosinophilic leukemoid is added.

In general, bone-marrow cells count did not show similar alterations to those found in the white blood cells count.

No relation was found between the increase in the number of leukocytes and the severity of the disease.

\section{BIBLIOGRAFÍA}

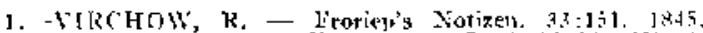

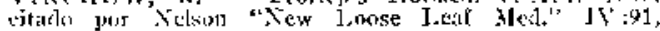
$1933^{-}$

2, ¿AI,BERT, A, y WELL, F. P. - Arti. Metl, Exp

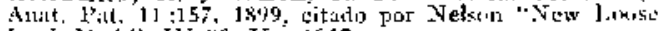

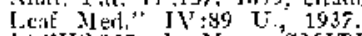

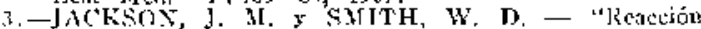

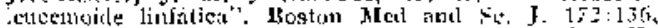
1915, citallo pur Nejon "New Luose Léti herl." IV: sy $\mathrm{r}, 1933$

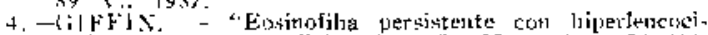

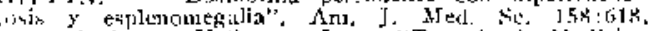

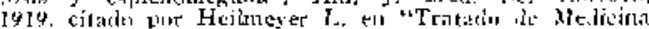
Internit" te fiergruitn, (i, von s stathelin, R. 2it.37.

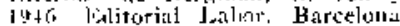

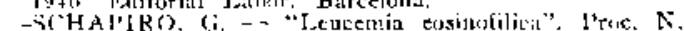

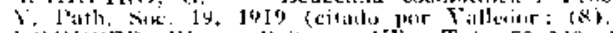

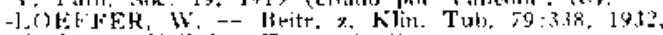
(ititl, pre: Villedtor, T, y col, (j)

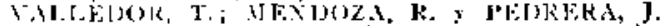

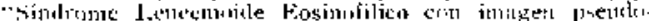

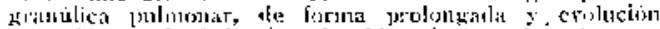

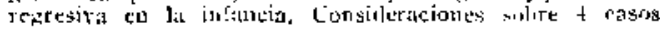




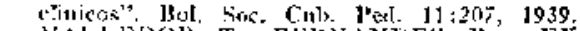

S.-YALALOR, T. FERNANEL\% 13, y EXPOSITO

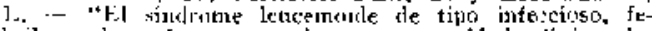

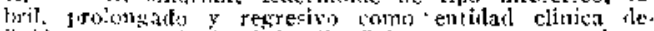

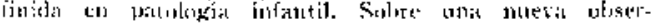

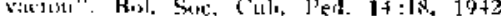

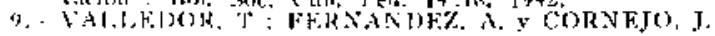

"Lusinofilis masiva de ligis lencemtinde, de prige:1 in-

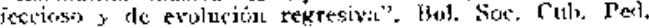
$15: 923,194,3$

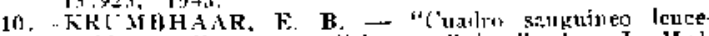

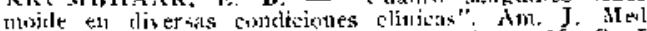
Sc. 172:514, 143ti, citado en Sew Orleats M, S. I. 99: $21,19+1$, 